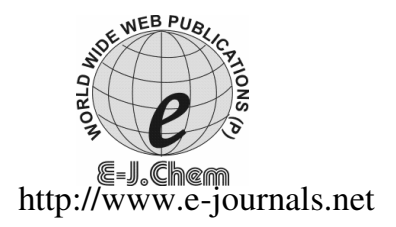

\author{
ISSN: 0973-4945; CODEN ECJHAO \\ E-Journal of Chemistry \\ 2011, 8(4), 1864-1871
}

\title{
Solvent Extraction Studies on Copper(II) and Silver(I) Complexes of Bis(4-hydroxypent-2-ylidene)diaminoethane: Composition of Extracted Copper(II) Species
}

\author{
P O UKOHA ${ }^{\S^{*}}$, F I. NWABUE and L N. OBASI ${ }^{\S}$ \\ ${ }^{\S}$ Department of Pure and Industrial Chemistry \\ University of Nigeria, Nsukka, Enugu State, Nigeria \\ Department of Industrial Chemistry \\ Ebonyi State University, Abakaliki, Ebonyi State, Nigeria \\ pius_ukoha@yahoo.com
}

Received 27 April 2010; Revised 7 November 2010; Accepted 18 November 2010

\begin{abstract}
Copper(II) complexes of bis(4-hydroxypent-2-ylidene)diaminoethane (BHPDE) were prepared by extractive method. Job's continuous variation and equilibrium constant methods, as well as spectral and elemental analyses were applied in the determination of the nature of extracted species. The results indicate the formation of mixed ionic complexes of the types $\mathrm{Cu}\left(\mathrm{H}_{2} \mathrm{~L}\right) \mathrm{X}_{2}$, $\mathrm{Cu}\left(\mathrm{H}_{2} \mathrm{~L}\right)_{2} \mathrm{X}_{2}$ and $\mathrm{CuL}$ (where $\mathrm{H}_{2} \mathrm{~L}=$ BHPDE and $\mathrm{X}=\mathrm{NO}_{3}^{-}, \mathrm{CIO}_{4}^{-}, \mathrm{Cl}^{-}$or $\left.1 / 2 \mathrm{SO}_{4}{ }^{2-}\right) . \mathrm{Cu}\left(\mathrm{H}_{2} \mathrm{~L}\right) \mathrm{X}_{2}$ and $\mathrm{Cu}\left(\mathrm{H}_{2} \mathrm{~L}\right)_{2} \mathrm{X}_{2}$ predominate at low $\mathrm{pH}$ extractions while $\mathrm{CuL}$ chelate predominates at high $\mathrm{pH}$ extraction. The complexes exist in two isomeric forms, the blue readily-water-soluble and the violet less water-soluble forms. Both complexes show maximum absorption at $540 \mathrm{~nm}$ and molar absorptivity values of about $2.0 \times 10^{2} \mathrm{dm}^{3} \mathrm{~mole}^{-1} \mathrm{~cm}^{-1}$.
\end{abstract}

Keywords: Extraction, Copper(II), Bis(4-hydroxypent-2-ylidene)diaminoethane, UV-Vis

\section{Introduction}

Bis(4-hydroxypent-2-ylidene)diaminoethane (BHPDE) has been synthesised and characterized via UV-Vis ${ }^{1,2}$, FTIR $^{3-5}$ and NMR $^{6,7}$. Through these means it was established to exist in three tautomeric forms ${ }^{8}$. The coordination chemistry of BHPDE with divalent metal ions and the potentials of BHPDE as a photometric reagent and as extractant for $\mathrm{Cu}(\mathrm{II})$ and many other metal ions have been documented ${ }^{9-12}$.

In our previous effort we had reported a comprehensive scheme for the extraction of $\mathrm{Cu}(\mathrm{II})$ and $\mathrm{Ag}(\mathrm{I})$ using BHPDE in various media ${ }^{13}$. Also we pursued an extensive study 
towards establishing the effect of medium $\mathrm{pH}$, complexing agents, acid concentration and salting-out agents on the extraction of the two metals ${ }^{14}$. A scheme for the separation of $\mathrm{Ag}(\mathrm{I})$ from $\mathrm{Cu}$ (II) in a synthetic mixture using BHPDE was proposed ${ }^{13,14}$.

The present effort is an attempt at characterizing the extracted $\mathrm{Cu}$ (II) species formed at the various conditions that afforded quantitative extraction. This is with the aim to gaining insight into the various extraction profiles presented by $\mathrm{Cu}(\mathrm{II})$ at high and low $\mathrm{pH}$ as well as in concentrated and dilute acid solutions.

\section{Experimental}

Bis(4-hydroxypent-2-ylidene)diaminoethane (BHPDE) was prepared as reported elsewhere ${ }^{13,14}$ and was characterized based on melting point, UV-Vis, IR and NMR spectra. Stock solutions of $\mathrm{Cu}$ (II) were prepared using $\mathrm{CuCl}_{2}$ (Merck, Analar grade). Buffer solutions were prepared following the Clark and Lubbs method ${ }^{15}$. Stock solutions of mineral acids $\left(\mathrm{HNO}_{3}\right.$, $\mathrm{HCl}, \mathrm{HClO}_{4}$ and $\mathrm{H}_{2} \mathrm{SO}_{4}$ ) were prepared by diluting the concentrated acids and were standardized using appropriate primary standard bases.

Electronic spectra of ligand and complexes were obtained on Pye-Unicam UV-Vis spectrophotometer. Infra-red spectra of the compounds were recorded using Thermo electron corporation IR 100 series. NMR data were run on Varian mercury YH200 MHz NMR spectrometer. Microanalysis of ligand and complexes was done at the Department of Chemistry, University of Sussex, England.

\section{Determination of composition of extracted species}

Job's continuous variation or equimolar method was employed in determining the composition of extracted species as reported elsewhere ${ }^{16}$ and confirmed by the method of equilibration constant ${ }^{17}$.

\section{Synthesis of $\mathrm{Cu}(\mathrm{II})$ - BHPDE complexes}

The complexes were synthesized from pre-determined high and low $\mathrm{pH}$ of quantitative extraction ${ }^{13,14}$ in order to ascertain the nature of extracted species. $\mathrm{CuCl}_{2}$ was dissolved in pH 9.0 buffer and evaporated to dryness and redissolved in the same buffer solution to obtain $20 \mathrm{~mL}$ of $0.001 \mathrm{M}$ metal ion solution. This solution was reacted with $2.23 \times 10^{-3}$ mole of freshly prepared BHPDE at $40{ }^{\circ} \mathrm{C}$ and stirred for $300 \mathrm{~s}$. The crystals formed were recrystallised in $\mathrm{H}_{2} \mathrm{O} /$ Dioxane (40/60), dried and characterized. The same procedure was followed in preparing the complex from low $\mathrm{pH}$ solution $\left(0.001\right.$ to $0.1 \mathrm{M} \mathrm{H}_{2} \mathrm{SO}_{4}, \mathrm{HNO}_{3}$, $\mathrm{HCl}$ and $\mathrm{HClO}_{4}$ ). The above procedures were repeated at room temperature and complexes formed extracted using $\mathrm{CHCl}_{3}$. The organic extracts were evaporated to dryness, the products recrystallised using $\mathrm{H}_{2} \mathrm{O}$ /dioxane, dried and characterized.

\section{Results and Discussion}

Job's continuous variation method was employed in investigating the metal-ligand mole ratio of copper(II)-BHPDE complexes extracted from acid solutions. The results as summarized in Figure 1 shows a metal-ligand mole ratio of 2:3 in all the acids studied. The result differs from what was obtained (1:1 metal-ligand ratio) in the log-log plot of BHPDE against distribution ratio (D) in Figure 2. This variation is likely due to the fact that at the conditions of extraction two types of complexes are formed having 1:1 and 1:2 metal-ligand ratio respectively. A combination of these gives a mixture of ionic complexes with 2:3 metal-ligand mole ratio especially at high ligand concentrations. Formation of ionic complexes of BHPDE with lanthanides ${ }^{18,19}$ and $z_{n i n c}{ }^{20}$ has been documented. Our results seem to be in conformity with these. 


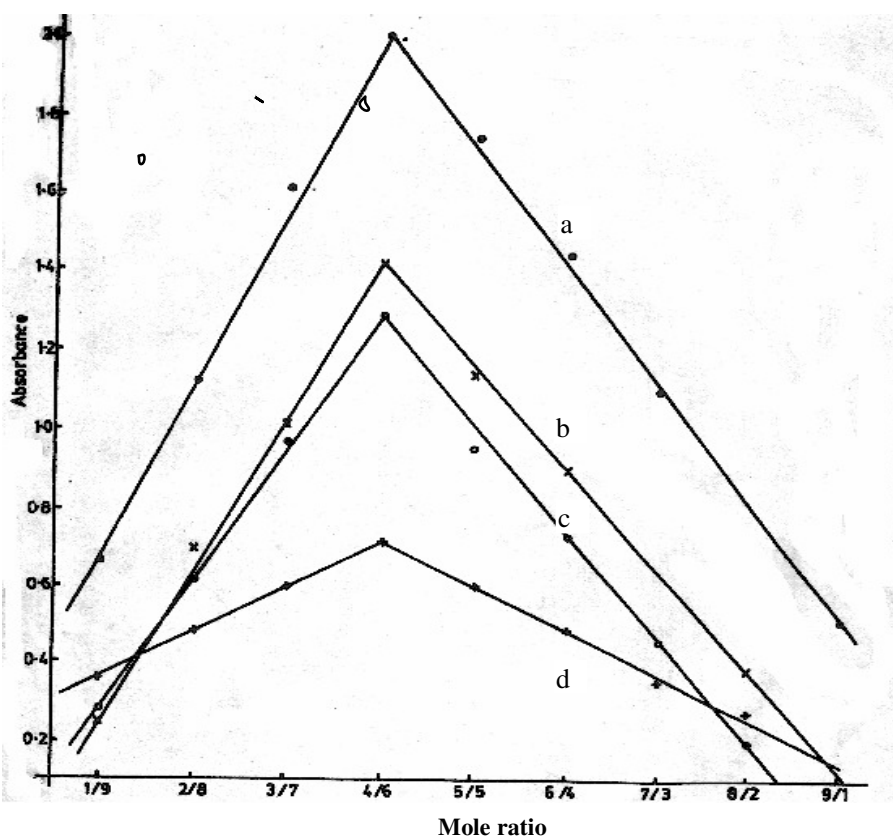

Figure 1. Jobs curve for $\mathrm{Cu}(\mathrm{II})-\mathrm{BHPDE}$ complex. Metal ligand mole ratio $=2: 3$. (a) $0.001 \mathrm{M} \mathrm{HCl}$; (b) $0.001 \mathrm{M} \mathrm{HNO}_{3}$; (c) $0.001 \mathrm{M} \mathrm{H}_{2} \mathrm{SO}_{4}$; (d) $0.001 \mathrm{M} \mathrm{HClO}_{4}$

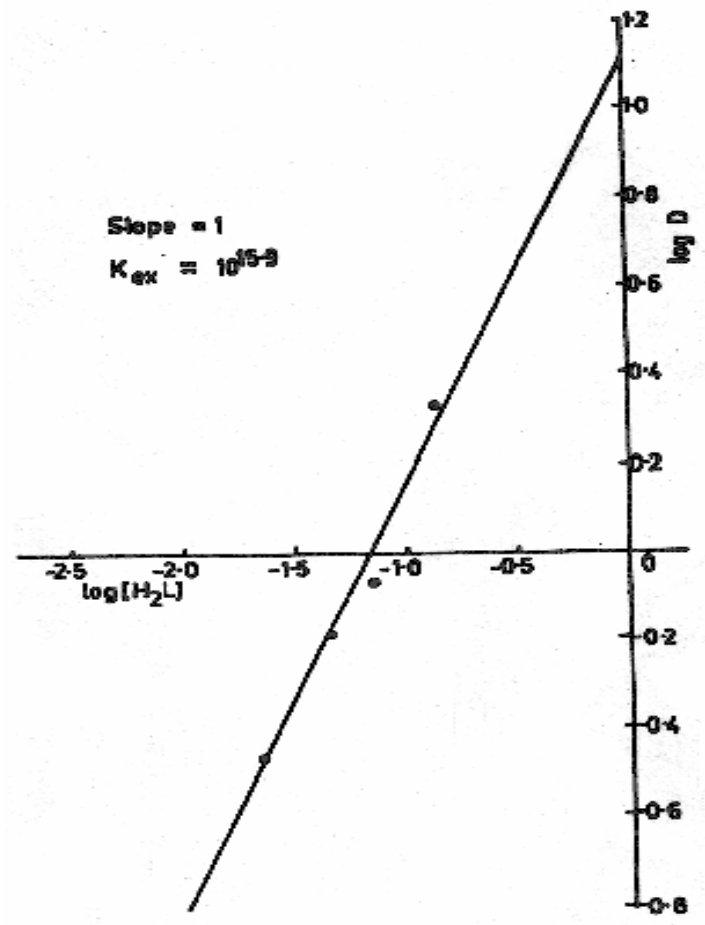

Figure 2. Effect of initial concentration of BHPDE on the distribution of $\mathrm{Cu}(\mathrm{II})$ 
The slope of the curve in Figure 2 gives the value of 1.0 implying 1:1 Cu-BHPDE mole ratio. This result is supported by the fact that at low $\mathrm{pH}\left(10^{-3}-10^{-1} \mathrm{M}\right.$, acid) the ligand is protonated and can complex with copper as a neutral molecule ${ }^{20-21}$. On the basis of this, extraction of copper(II) could be explained using the following equations.

$$
\begin{aligned}
{\left[\mathrm{Cu}\left(\mathrm{H}_{2} \mathrm{O}\right)_{6}\right]^{2+}+2 \mathrm{H}_{3} \mathrm{~B}^{+}+2 \mathrm{X}^{-} } & \rightleftharpoons \mathrm{Cu}\left(\mathrm{H}_{2} \mathrm{~B}\right)_{2} \mathrm{X}_{2}+2 \mathrm{H}^{+}+6 \mathrm{H}_{2} \mathrm{O} \\
\mathrm{Kex} & =\frac{\left[\mathrm{Cu}\left(\mathrm{H}_{2} \mathrm{~B}\right)_{2} \cdot \mathrm{X}_{2}\right] \cdot\left[\mathrm{H}^{+}\right]^{2}}{\left[\mathrm{Cu}\left(\mathrm{H}_{2} \mathrm{O}\right)_{6}^{2+}\right] \cdot\left[\mathrm{H}_{3} \mathrm{~B}^{+}\right]^{2} \cdot\left[\mathrm{X}^{-}\right]^{2}} \\
& =\mathrm{D} \times \frac{\left[\mathrm{H}^{+}\right]^{2}}{\left[\mathrm{X}^{-}\right]^{2}\left[\mathrm{H}_{3} \mathrm{~B}^{+}\right]^{2}}
\end{aligned}
$$

$($ Kex $=$ extraction constant; $\mathrm{D}=$ distribution ratio $)$. As reported previously ${ }^{14,15}$, the first base dissociation constant $\mathrm{K}_{1 \mathrm{~b}}$ is given as

$$
\begin{aligned}
\mathrm{K}_{1 \mathrm{~b}} & =\frac{\left[\mathrm{H}_{3} B^{+}\right]}{\left[\mathrm{H}_{2} B\right]\left[H^{+}\right]} \\
\therefore \quad\left[\mathrm{H}_{3} \mathrm{~B}^{+}\right] & =\mathrm{K}_{1 \mathrm{~b}}\left[\mathrm{H}_{2} \mathrm{~B}\right]\left[\mathrm{H}^{+}\right]
\end{aligned}
$$

Substituting equation (4) into (3) gives

$$
\begin{aligned}
\operatorname{Kex} & =\frac{D}{\left[\mathrm{X}^{-}\right]^{2} \mathrm{Kib}^{2}\left[\mathrm{H}_{2} \mathrm{~B}\right]^{2}} \\
\therefore \log \mathrm{Kex} & =\log \mathrm{D}-2 \log \left[\mathrm{X}^{-}\right]-2 \log \left[\mathrm{H}_{2} \mathrm{~B}\right]-2 \log \mathrm{K}_{1 \mathrm{~b}} \\
\therefore \log \mathrm{D} & =2 \log \left[\mathrm{X}^{-}\right]+2 \log \left[\mathrm{H}_{2} \mathrm{~B}\right]+2 \log \mathrm{K}_{1 \mathrm{~b}}+\log \mathrm{Kex} \\
\left(\mathrm{H}_{2} \mathrm{~B}\right. & \left.=\mathrm{BHPDE} \text { and } \mathrm{X}=\mathrm{NO}_{3}, \mathrm{Cl}, \text { or }{ }^{1 / 2} \mathrm{SO}_{4}\right)
\end{aligned}
$$

The determined extraction constant, Kex, from equation (7) as depicted in Figure 2 is $10^{15.9}$. The ligand behaves as a weak acid and in high $\mathrm{pH}$ media ${ }^{13,14}$ formation of a chelate will probably be according to equation (8).

$$
\left[\mathrm{Cu}\left(\mathrm{H}_{2} \mathrm{O}\right)_{6}\right]^{2+}+\mathrm{H}_{2} \mathrm{~B} \rightleftharpoons \mathrm{CuB}+2 \mathrm{H}^{+}
$$

\section{Molecular formulae of complexes}

Table 1 shows some physical properties and microanalytical data of both ligand and complexes. Complexes prepared in high $\mathrm{pH}$ media show sharp melting point $\left(\approx 145{ }^{\circ} \mathrm{C}\right)$ while those prepared at low $\mathrm{pH}$ decompose at about $240{ }^{\circ} \mathrm{C}$. This indicates that at high $\mathrm{pH}$, 1:1 complexes of the form $\mathrm{CuB}$ are formed and their nature is not dependent on whether they are extracted or synthesized.

At low $\mathrm{pH}$ of the aqueous mixture the complexes formed are either 1:2 or 2:3 metal-ligand ratio. The 2:3 complexes being a mixture of $1: 1$ and $1: 2$ as stated above. The complexes formed at high $\mathrm{pH}$ are mostly greenish or violet whereas at low $\mathrm{pH}$, violet and blue (purple) complexes are obtained. The violet form predominates in organic solvents and is the most stable. Other forms tend to give violet colour in organic solvents. The violet form crystallizes more readily from aqueous preparation media than the blue form.

From Table 1, it is also noticeable that the number of anions attached to the complex seems to depend on the anion concentration. This is quite evident in the complexes prepared in $\mathrm{HCl}$ media. 
Table 1. Physical and microanalytical data of ligand and complexes

\begin{tabular}{|c|c|c|c|c|c|c|c|c|c|c|}
\hline \multirow{2}{*}{$\begin{array}{l}\text { Molecular } \\
\text { formula }\end{array}$} & \multirow{2}{*}{$\begin{array}{l}\text { preparation } \\
\text { Condition }\end{array}$} & \multirow{2}{*}{ m.p, ${ }^{\circ} \mathrm{C}$} & \multirow{2}{*}{$\begin{array}{c}\text { Yield, } \\
\%\end{array}$} & \multirow{2}{*}{ Colour- } & \multicolumn{3}{|c|}{ Required, $\%$} & \multicolumn{3}{|c|}{ Found, $\%$} \\
\hline & & & & & $\mathrm{C}$ & $\mathrm{H}$ & $\mathrm{N}$ & $\mathrm{C}$ & $\mathrm{H}$ & $\mathrm{N}$ \\
\hline $\mathrm{C}_{12} \mathrm{H}_{20} \mathrm{~N}_{2} \mathrm{O}_{2}$ & - & $111-112$ & 95.5 & White & 64.24 & 8.93 & 12.50 & 63.98 & 7.45 & 12.43 \\
\hline $\mathrm{CuC}_{12} \mathrm{H}_{18} \mathrm{~N}_{2} \mathrm{O}_{2}$ & pH 9 (Ext) & $114-112$ & 56 & Violet & 50.41 & 7.03 & 9.80 & 50.15 & 6.18 & 9.32 \\
\hline $\mathrm{CuC}_{12} \mathrm{H}_{18} \mathrm{~N}_{2} \mathrm{O}_{2}$ & pH 9 (Synth) & 146 & 48 & Violet & 50.41 & 7.03 & 9.80 & 49.74 & 6.15 & 9.36 \\
\hline $\begin{array}{c}\mathrm{Cu}\left(\mathrm{C}_{12} \mathrm{H}_{20} \mathrm{~N}_{2} \mathrm{O}_{2}\right)_{3} \\
\mathrm{Cl}_{3}(\mathrm{OH})\end{array}$ & $\begin{array}{c}10^{-3} \mathrm{M} \mathrm{HCl} \\
(\text { Synth })\end{array}$ & 239(Dec) & 62 & Purple & 49.42 & 6.93 & 9.60 & 49.74 & 6.15 & 7.32 \\
\hline $\begin{array}{c}\mathrm{Cu}_{2}\left(\mathrm{C}_{12} \mathrm{H}_{20} \mathrm{~N}_{2} \mathrm{O}_{2}\right)_{3} \\
\mathrm{Cl}_{3}(\mathrm{OH})\end{array}$ & $\begin{array}{c}10^{-3} \mathrm{M} \mathrm{HCl} \\
(\mathrm{Ext})\end{array}$ & 240 (Dec) & 59 & Purple & 46.81 & 6.67 & 9.10 & 46.02 & 5.67 & Not det \\
\hline $\begin{array}{c}\mathrm{Cu}\left(\mathrm{C}_{12} \mathrm{H}_{20} \mathrm{~N}_{2} \mathrm{O}_{2}\right)_{2} \\
\left(\mathrm{NO}_{3}\right)(\mathrm{OH})\end{array}$ & $\begin{array}{l}10^{-3} \mathrm{M} \mathrm{HNO}_{3} \\
\text { (Ext) }\end{array}$ & 235(Dec) & 43 & Blue & 48.75 & 7.001 & 11.84 & 48.41 & 6.69 & Not det \\
\hline $\begin{array}{c}\mathrm{Cu}\left(\mathrm{C}_{12} \mathrm{H}_{20} \mathrm{~N}_{2} \mathrm{O}_{2}\right)_{2} \\
\left(\mathrm{NO}_{3}\right)(\mathrm{OH})\end{array}$ & $\begin{array}{l}10^{-2} \mathrm{M} \mathrm{HNO}_{3} \\
\text { (Ext) }\end{array}$ & 235(Dec) & 50 & Blue & 48.75 & 7.001 & 11.84 & 47.88 & 6.63 & Not det \\
\hline $\begin{array}{c}\mathrm{Cu}_{2}\left(\mathrm{C}_{12} \mathrm{H}_{20} \mathrm{~N}_{2} \mathrm{O}_{2}\right)_{3} \\
\mathrm{Cl}_{2} \mathrm{SO}_{4}\end{array}$ & $\begin{array}{c}10^{-3} \mathrm{M} \mathrm{H}_{2} \mathrm{SO}_{4} \\
\text { (Synth) }\end{array}$ & 236(Dec) & 45.9 & Violet & 44.7 & 6.30 & 8.69 & 45.71 & 5.25 & Not det \\
\hline $\begin{array}{c}\mathrm{Cu}_{2}\left(\mathrm{C}_{12} \mathrm{H}_{20} \mathrm{~N}_{2} \mathrm{O}_{2}\right)_{3} \\
\mathrm{Cl}_{2}(\mathrm{OH})_{2}\end{array}$ & $\begin{array}{c}10^{-3} \mathrm{M} \mathrm{H}_{2} \mathrm{SO}_{4} \\
(\mathrm{Ext})\end{array}$ & 236(Dec) & 42.3 & Violet & 47.8 & 6.92 & 9.30 & 47.68 & 6.06 & 8.65 \\
\hline $\begin{array}{c}\mathrm{Cu}_{2}\left(\mathrm{C}_{12} \mathrm{H}_{20} \mathrm{~N}_{2} \mathrm{O}_{2}\right)_{3} \\
\mathrm{Cl}_{2}(\mathrm{OH})_{2}\end{array}$ & $\begin{array}{c}10^{-3} \mathrm{M} \mathrm{HClO}_{4} \\
\text { (Ext) }\end{array}$ & 235(De & 47.2 & Violet & 47.8 & 6.92 & 9.30 & 47.88 & 5.43 & 8.83 \\
\hline $\begin{array}{c}\mathrm{Cu}_{2}\left(\mathrm{C}_{12} \mathrm{H}_{20} \mathrm{~N}_{2} \mathrm{O}_{2}\right)_{3} \\
\mathrm{Cl}_{3}(\mathrm{OH})_{2}\end{array}$ & $\begin{array}{c}10^{-3} \mathrm{M} \mathrm{HClO}_{4} \\
\text { (Synth) }\end{array}$ & 235(Dec) & 46.0 & Purple & 47.8 & 6.92 & 9.30 & 47.11 & 5.19 & 9.00 \\
\hline
\end{tabular}

Legend: Ext $=$ Extracted $;$ Synth $=$ Synthesized $;$ Dec $=$ Decomposes $;$ Not det $=$ Not determined

\section{Electronic spectra}

UV spectral data of BHPDE and its synthesized and extracted $\mathrm{Cu}$ (II) complexes are displayed in Table 2. The ligand absorbs at $321.9 \mathrm{~nm}$ with a shoulder at $237 \mathrm{~nm}$ and molar absorptivities are $5.3 \times 10^{4}$ and $1.1 \times 10^{4} \mathrm{~L} \mathrm{~mol}^{-1} \mathrm{~cm}^{-1}$ respectively. This has been attributed to $\mathrm{n} \rightarrow \pi^{*}$ and $\pi \rightarrow \pi^{*}$ transitions. The complexes absorb between 297 and $310 \mathrm{~nm}$ with molar extinction between $10^{4}$ to $10^{6} \mathrm{~L} \mathrm{~mol}^{-1} \mathrm{~cm}^{-1}$. Another prominent peak at about $241 \mathrm{~nm}$ also appears in the complexes extracted from high $\mathrm{pH}$ media. From what has been reported for other Schiff base complexes ${ }^{18}$ the peak at $321.9 \mathrm{~nm}$ in the ligand and now at $297 \mathrm{~nm}$ and $310 \mathrm{~nm}$ in the complexes is due to electronic transition in the hydrogen-bonded chelate rings. Hypsochromic shift in the complexes is likely the result of interaction of the metal ion with the non-bonding electrons of ketoamine nitrogens.

The visible spectra of complexes (Table 2) present a maximum absorption at $540 \mathrm{~nm}$ and molar absorptivity of about $10^{2}$. Although three bands should be expected for tetragonally distorted $\mathrm{Cu}$ (II) complexes for the transitions ${ }^{2} \mathrm{~B}_{1 \mathrm{~g}} \rightarrow{ }^{2} \mathrm{~A}_{2},{ }^{2} \mathrm{~B}_{1 \mathrm{~g}} \rightarrow{ }^{2} \mathrm{~B}_{2 \mathrm{~g}}$, and ${ }^{2} \mathrm{~B}_{1 \mathrm{~g}}$ $\rightarrow 2 \mathrm{E}_{\mathrm{g}}$ in order to increasing energy, but in general one broad band is obtained with or without shoulder at about $1400-800 \mathrm{~cm}^{-1}$. This is likely due to $\mathrm{d} \leftarrow \mathrm{d}$ transitions or $\mathrm{d} \leftarrow \mathrm{d}$ transitions coupled with charge transfer spectra ${ }^{18}$. Table 3 shows that the complexes formed under various conditions absorbed between $540 \mathrm{~nm}$ to $545 \mathrm{~nm}$ with molar absorptivities of 200 to $252 \mathrm{~L} \mathrm{~mol}^{-1} \mathrm{~cm}^{-1}$ confirming the fact that the mode of preparation (extracted or synthesized) did not confer extra properties on the complexes. 
Table 2. Ultra-Violet spectra of BHPDE and its copper(II) complexes in chloroform

\begin{tabular}{|c|c|c|c|c|c|c|c|}
\hline $\begin{array}{l}\text { Molecular } \\
\text { formula }\end{array}$ & $\begin{array}{l}\text { Preparation } \\
\text { Condition }\end{array}$ & $\begin{array}{c}\lambda_{\max } 1 \\
n m\end{array}$ & $\begin{array}{c}\varepsilon_{\max } 1, \\
\mathrm{~L} \mathrm{~mole}{ }^{-1} \\
\mathrm{~cm}^{-1}\end{array}$ & $\begin{array}{c}\lambda_{\max } 2 \\
n m\end{array}$ & $\begin{array}{c}\varepsilon_{\max } 2, \\
\mathrm{~L} \mathrm{~mole}^{-1} \\
\mathrm{~cm}^{-1}\end{array}$ & $\begin{array}{c}\lambda_{\max } 3 \\
n m\end{array}$ & $\begin{array}{c}\varepsilon_{\max } 3, \\
\mathrm{~L} \mathrm{~mole}^{-1} \\
\mathrm{~cm}^{-1}\end{array}$ \\
\hline BHPDE & - & $321.9(\mathrm{~s})$ & $5.3 \times 10^{4}$ & $327(\mathrm{sh})$ & $1.1 \times 10^{4}$ & - & - \\
\hline $\mathrm{Cu} B$ & pH 9 (Ext) & 311.6(s) & $4.9 \times 10^{4}$ & $272.5(b)$ & $4.8 \times 10^{4}$ & $241.9(\mathrm{sh})$ & $4.3 \times 10^{4}$ \\
\hline $\mathrm{Cu} \mathrm{B}$ & pH 9 (Synth) & 311.7(s) & $2.0 \times 10^{4}$ & $277.2(\mathrm{sh})$ & $1.0 \times 10^{4}$ & $240.7(\mathrm{~s})$ & $1.4 \times 10^{4}$ \\
\hline $\mathrm{Cu}\left(\mathrm{H}_{2} \mathrm{~B}\right)_{2} \cdot \mathrm{Cl}_{2}$ & $\begin{array}{c}10^{-5} \mathrm{M} \mathrm{HCl} \\
\text { (Synth) }\end{array}$ & 310.9(s) & $2.1 \times 10^{5}$ & 242.7(s) & $1.4 \times 10^{5}$ & - & - \\
\hline $\begin{array}{c}\mathrm{Cu}_{2}\left(\mathrm{H}_{2} \mathrm{~B}\right)_{3} . \\
\mathrm{Cl}_{2}(\mathrm{OH})\end{array}$ & $\begin{array}{c}10^{-2} \mathrm{M} \mathrm{HCl} \\
(\text { Ext) }\end{array}$ & 297.3(s) & $8.3 \times 10^{4}$ & $245(s)$ & $6.7 \times 10^{4}$ & - & - \\
\hline $\begin{array}{l}\mathrm{Cu}\left(\mathrm{H}_{2} \mathrm{~B}\right)_{2} . \\
\mathrm{NO}_{3} \cdot(\mathrm{OH})\end{array}$ & $\begin{array}{c}10^{-3} \mathrm{M} \mathrm{HNO}_{3} \\
\text { (Ext) }\end{array}$ & $310.4(\mathrm{~s})$ & $6.8 \times 10^{4}$ & 243.5(s) & $5.6 \times 10^{4}$ & - & - \\
\hline $\begin{array}{l}\mathrm{Cu}\left(\mathrm{H}_{2} \mathrm{~B}\right)_{2} . \\
\mathrm{NO}_{3}(\mathrm{OH})\end{array}$ & $\begin{array}{c}10^{-2} \mathrm{M} \\
\mathrm{HNO}_{3}(\text { Synth })\end{array}$ & 307.4(s) & $1.5 \times 10^{4}$ & 242.4(s) & $1.2 \times 10^{4}$ & - & - \\
\hline $\begin{array}{l}\mathrm{Cu}_{2}\left(\mathrm{H}_{2} \mathrm{~B}\right)_{3} . \\
\mathrm{Cl}_{2} .(\mathrm{OH})_{2}\end{array}$ & $\begin{array}{c}10^{-3} \mathrm{M} \mathrm{H}_{2} \mathrm{SO}_{4} \\
\text { (Ext) }\end{array}$ & $296.8(\mathrm{~s})$ & $8.9 \times 10^{4}$ & 246.4(s) & $7.6 \times 10^{4}$ & - & - \\
\hline $\begin{array}{c}\mathrm{Cu}_{2}\left(\mathrm{H}_{2} \mathrm{~B}\right)_{3} . \\
\mathrm{Cl}_{2} \cdot \mathrm{SO}_{4}\end{array}$ & $\begin{array}{c}10^{-1} \mathrm{M} \mathrm{H}_{2} \mathrm{SO}_{4} \\
\text { (Synth) }\end{array}$ & $309.6(s)$ & $5.4 \times 10^{4}$ & 243.1(s) & $4.1 \times 10^{4}$ & - & - \\
\hline $\begin{array}{l}\mathrm{Cu}_{2}\left(\mathrm{H}_{2} \mathrm{~B}\right)_{3} . \\
\mathrm{Cl}_{2} .(\mathrm{OH})_{2}\end{array}$ & $\begin{array}{c}10^{-3} \mathrm{M} \mathrm{HClO}_{4} \\
\text { (Synth) }\end{array}$ & 298.1(s) & $2.0 \times 10^{5}$ & 246.4(s) & $1.3 \times 10^{5}$ & - & - \\
\hline $\begin{array}{l}\mathrm{Cu}_{2}\left(\mathrm{H}_{2} \mathrm{~B}\right)_{3} . \\
\mathrm{Cl}_{2} .(\mathrm{OH})_{2}\end{array}$ & $\begin{array}{c}10^{-3} \mathrm{M} \mathrm{HClO}_{4} \\
\text { (Ext) }\end{array}$ & 296.8(s) & $1.1 \times 10^{6}$ & 247.5(s) & $8.9 \times 10^{4}$ & - & - \\
\hline
\end{tabular}

Legend: $S=$ Sharp; Sh = Shoulder; B =Broad; Ext = Extracted; Synth $=$ Synthesized

Table 3. Visible spectra of some of Cu-BHPDE complexes in chloroform

\begin{tabular}{cccc}
\hline Molecular formula & Method of Preparation & $\lambda_{\max }, \mathrm{nm}$ & $\varepsilon_{\max } 2, \mathrm{~L} \mathrm{~mole}^{-1} \mathrm{~cm}^{-1}$ \\
\hline $\mathrm{Cu} \mathrm{B}$ & $\mathrm{pH} 9($ Ext $)$ & 540 & 200.1 \\
$\mathrm{Cu} \mathrm{B}$ & $\mathrm{pH} 9$ (Synth) & 543 & 202.3 \\
$\mathrm{Cu}_{2}\left(\mathrm{H}_{2} \mathrm{~B}\right)_{3} \cdot \mathrm{Cl}_{2} \cdot \mathrm{SO}_{4}$ & $10^{-1} \mathrm{M} \mathrm{H}_{2} \mathrm{SO}_{4}$ (Synth) & 541 & 240 \\
$\mathrm{Cu}_{2}\left(\mathrm{H}_{2} \mathrm{~B}\right)_{3} \cdot \mathrm{Cl}_{2}(\mathrm{OH})_{2}$ & $10^{-2} \mathrm{M} \mathrm{HClO}_{4}$ (Ext) & 545 & 252 \\
\hline \multicolumn{4}{c}{ Legend: $\mathrm{H}_{2} B=$ BHPDE; Ext $=$ Extracted; Synth $=$ Synthesized }
\end{tabular}

\section{FTIR spectra}

Table 4 gives the essential peaks of ligand and complexes and presents a scheme for determining the mode of ligation of the ligand. The peak at $3100 \mathrm{~cm}^{-1}$ due to hydrogen-bonded $\mathrm{OH}$ or $\mathrm{NH}$ in the ligand appears with weaker intensities at $2900 \mathrm{~cm}^{-1}$ in complexes prepared from low $\mathrm{pH}$ but is absent in complexes prepared from high $\mathrm{pH}$ media. This is indicative of ligation to the nitrogen and oxygen atoms of the undissociated ligand at low $\mathrm{pH},-\mathrm{NH}$ and $-\mathrm{OH}$ vibrations being limited by the presence of the metal. At high $\mathrm{pH}$ deprotonation takes place before complexation thereby leading to absence of $v(\mathrm{NH})$ or $v(\mathrm{OH})$.

Free $\mathrm{OH}$ peaks at $3550 \mathrm{~cm}^{-1}$ in the complexes extracted and prepared from $\mathrm{pH} 9$ and $10^{-3} \mathrm{M} \mathrm{HCl}$ is not likely due to coordinated water since it is not supported by elemental analysis. It is probably due to presence of moisture in the Nujol mulls. The bands at $1600-1645 \mathrm{~cm}^{-1}$ in both ligand and complexes extracted and synthesized from $\mathrm{pH} 9$ is likely due to hydrogen-bonded carbonyl stretch. On chelation the carbonyl stretch suffers only slight distortion as only the imino hydrogens are removed before chelation. The band between $1600-1560 \mathrm{~cm}^{-1}$ is ascribed to $v(\mathrm{C}=\mathrm{C})$ and $1560-1430 \mathrm{~cm}^{-1}$ due to $v(\mathrm{C}=\mathrm{N})$. The lower frequencies of some of these bands is due to intramolecular hydrogen-bonding. 
Table 4. Infrared Absorption Bands $\left(\mathrm{cm}^{-1}\right)$ of Nujol Mulls

\begin{tabular}{|c|c|c|c|c|c|c|c|c|c|c|c|c|}
\hline Assignment & $\begin{array}{c}-\mathrm{OH} \text {, from } \\
\text { water }\end{array}$ & $\mathrm{OH}$ & $\mathrm{NH}$ & $\begin{array}{l}\mathrm{CH}_{3}, \\
\mathrm{CH}_{2} \\
\end{array}$ & $\mathrm{C}=\mathrm{O}$ & $\mathrm{C}=\mathrm{C}$ & $\mathrm{C}=\mathrm{N}$ & $\mathrm{C}-\mathrm{CH}_{3}$ & $\mathrm{C}-\mathrm{N}$ & $\mathrm{C}-\mathrm{O}$ & $\mathrm{C}=\mathrm{C}-\mathrm{H}$ & $\mathrm{Cu}-\mathrm{O}$ \\
\hline BHPDE & - & $\begin{array}{l}3100 \\
\text { (br) }\end{array}$ & 2900 (br) & $2820(w)$ & $1625(w)$ & $1590(\mathrm{br})$ & $1500(\mathrm{~s})$ & $\begin{array}{l}\text { 1440(w) } \\
1355(w)\end{array}$ & $1340(s)$ & $\begin{array}{l}1270(\mathrm{~s}) \\
1125(\mathrm{w})\end{array}$ & $920(w)$ & \\
\hline Cu B. pH 9 (ext) & 3500 (br) & & $2890(w)$ & $2820(w)$ & $1630(w)$ & $1525(\mathrm{~m})$ & $1490(w)$ & $\begin{array}{l}1440(\mathrm{br}) \\
1360(\mathrm{br})\end{array}$ & $1335(w)$ & $\begin{array}{l}1260(w) \\
1100(w)\end{array}$ & $930(s)$ & $235(w)$ \\
\hline $\begin{array}{c}\mathrm{Cu} \text { B. pH } 9 \\
\text { (synth) }\end{array}$ & 3500 (br) & & $2890(w)$ & 2820 (br) & $1640(w)$ & $1570(w)$ & $1500(w)$ & $\begin{array}{l}1450(\mathrm{w}) \\
1400(\mathrm{br})\end{array}$ & $1335(\mathrm{br})$ & $\begin{array}{l}1250(w) \\
1100(\mathrm{~s})\end{array}$ & $\begin{array}{l}930(s) \\
300(s)\end{array}$ & $265(w)$ \\
\hline $\begin{array}{c}\left(\mathrm{Cu}\left(\mathrm{H}_{2} \mathrm{~B}\right)_{2} \cdot \mathrm{Cl}_{2}\right) \\
10^{-2} \mathrm{M} \mathrm{HCl} \text { (synth) }\end{array}$ & 3500 (br) & & $2900(w)$ & $2820(w)$ & & $1560(w)$ & $1500(w)$ & 1400 & $1335(\mathrm{w})$ & $\begin{array}{c}1260(\mathrm{~m}) \\
1100(\mathrm{~s})\end{array}$ & $\begin{array}{l}930(\mathrm{vs}) \\
280(\mathrm{~m})\end{array}$ & $200(w)$ \\
\hline $\begin{array}{c}\left(\mathrm{Cu}\left(\mathrm{H}_{2} \mathrm{~B}\right)_{3} \cdot \mathrm{Cl}_{2}\right. \\
(\mathrm{OH})) 10^{-2} \mathrm{M} \mathrm{HCl} \\
\text { (synth) }\end{array}$ & & & $2900(\mathrm{~m})$ & $2820(w)$ & & $1560(\mathrm{~m})$ & $1510(\mathrm{~m})$ & $\begin{array}{l}1440(\mathrm{~m}) \\
1395(\mathrm{w})\end{array}$ & $1340(w)$ & $\begin{array}{l}1260(\mathrm{~s}) \\
1175(\mathrm{w})\end{array}$ & $\begin{array}{l}920(\mathrm{~s}) \\
270(\mathrm{~m})\end{array}$ & $250(w)$ \\
\hline $\begin{array}{c}\left(\mathrm{Cu}\left(\mathrm{H}_{2} \mathrm{~B}\right)_{2} \cdot \mathrm{NO}_{3}\right. \\
(\mathrm{OH})) 10^{-3} \mathrm{M} \\
\mathrm{HNO}_{3}(\mathrm{ext})\end{array}$ & & & $2890(\mathrm{~s})$ & $2820(w)$ & & $1560(\mathrm{~s})$ & $1510(\mathrm{~m})$ & $\begin{array}{l}1440(w) \\
1360(w)\end{array}$ & $1340(w)$ & $\begin{array}{l}1260(\mathrm{~s}) \\
1100(\mathrm{w})\end{array}$ & $\begin{array}{l}920(\mathrm{~m}) \\
270(\mathrm{~m})\end{array}$ & $200(w)$ \\
\hline $\begin{array}{c}\left(\mathrm{Cu}\left(\mathrm{H}_{2} \mathrm{~B}\right)_{2} \cdot \mathrm{NO}_{3}\right. \\
(\mathrm{OH})) 10^{-3} \mathrm{M} \\
\mathrm{HNO}_{3}(\mathrm{ext})\end{array}$ & & & 2895(vs) & $2820(\mathrm{~s})$ & & $1560(\mathrm{~s})$ & $1510(\mathrm{~m})$ & $\begin{array}{l}1440(\mathrm{~m}) \\
1360(\mathrm{w})\end{array}$ & $1340(w)$ & $\begin{array}{l}1260(\mathrm{~s}) \\
1100(\mathrm{w})\end{array}$ & $\begin{array}{l}920(\mathrm{~m}) \\
275(\mathrm{~m})\end{array}$ & 200 \\
\hline $\begin{array}{c}\left(\mathrm{Cu}_{2}\left(\mathrm{H}_{2} \mathrm{~B}\right)_{3} \cdot \mathrm{Cl}_{2} \mathrm{~S}\right. \\
\left.\left.\mathrm{O}_{4}\right)\right) 10^{-1} \mathrm{M} \\
\mathrm{H}_{2} \mathrm{SO}_{4} \text { (synth) }\end{array}$ & & & 2895(br) & $2820(\mathrm{~s})$ & & $1560(\mathrm{~s})$ & $1510(\mathrm{~m})$ & $\begin{array}{l}1440(\mathrm{~m}) \\
1360(\mathrm{w})\end{array}$ & $1340(w)$ & $\begin{array}{l}1260(\mathrm{~s}) \\
1100(\mathrm{w})\end{array}$ & $\begin{array}{l}920(\mathrm{~s}) \\
280(\mathrm{~s})\end{array}$ & $299(\mathrm{~m})$ \\
\hline $\begin{array}{c}\left(\mathrm{Cu}_{2}\left(\mathrm{H}_{2} \mathrm{~B}\right)_{2} \cdot \mathrm{Cl}_{2}\right. \\
\left.(\mathrm{OH})_{2}\right) 10^{-2} \mathrm{M} \\
\mathrm{H}_{2} \mathrm{SO}_{4}(\mathrm{ext})\end{array}$ & & & 2900(br) & $2820(\mathrm{~s})$ & & $1560(w)$ & $1510(w)$ & $\begin{array}{l}1440(\mathrm{~m}) \\
1400(\mathrm{w})\end{array}$ & $1340(w)$ & $\begin{array}{l}1260(\mathrm{~s}) \\
1200(\mathrm{w})\end{array}$ & $\begin{array}{l}925(\mathrm{~m}) \\
270(\mathrm{~s})\end{array}$ & $200(\mathrm{~m})$ \\
\hline $\begin{array}{c}\left(\mathrm{Cu}_{2}\left(\mathrm{H}_{2} \mathrm{~B}\right)_{3} \cdot \mathrm{Cl}_{2}\right. \\
\left.(\mathrm{OH})_{2}\right) 10^{-3} \mathrm{M} \\
\mathrm{HClO}_{4}(\mathrm{ext})\end{array}$ & & & 2895(br) & $2820(\mathrm{~s})$ & & $1560(\mathrm{~m})$ & & $\begin{array}{l}1440(\mathrm{~m}) \\
1360(\mathrm{w})\end{array}$ & $1340(\mathrm{w})$ & $1260(s)$ & $\begin{array}{l}920(\mathrm{~m}) \\
275(\mathrm{~m})\end{array}$ & $200(w)$ \\
\hline $\begin{array}{c}\left(\mathrm{Cu}_{2}\left(\mathrm{H}_{2} \mathrm{~B}\right)_{3} \cdot \mathrm{Cl}_{2}\right. \\
\left.(\mathrm{OH})_{2}\right) 10^{-3} \mathrm{M} \\
\mathrm{HClO}_{4} \text { (synth) }\end{array}$ & & & $2900(\mathrm{~m})$ & $2820(w)$ & & $1560(\mathrm{~m})$ & $1510(\mathrm{~m})$ & $\begin{array}{l}1440(\mathrm{~m}) \\
1395(\mathrm{w})\end{array}$ & $1360(w)$ & $\begin{array}{l}1260(\mathrm{~s}) \\
1170(\mathrm{~m})\end{array}$ & $\begin{array}{l}920(\mathrm{~s}) \\
280(\mathrm{~m})\end{array}$ & $200(w)$ \\
\hline
\end{tabular}


$\mathrm{NO}_{3}$ vibrational mode appeared at 1360 and $700 \mathrm{~cm}^{-1}$ for complexes prepared in $\mathrm{HNO}_{3}$. Presence of absorption bands in the far IR between 440 and $250 \mathrm{~cm}^{-1}$ are due to $\mathrm{Cu}-\mathrm{O}$ and $\mathrm{Cu}-\mathrm{N}$ vibrations. $\mathrm{C}-\mathrm{O}$ stretching frequencies between $1150-1020 \mathrm{~cm}^{-1}$ are apparent in the ligand and in the complexes in high $\mathrm{pH}$ medium.

\section{NMR Data}

Proton NMR data of the ligand in $\mathrm{CDCl}_{3}$ with TMS as internal reference is on Table 5. The sharp doublet at 1.87 integrated for 12 protons is assigned to the slightly non-equivalent $\mathrm{CH}_{3} \mathrm{C}-\mathrm{N}$ and $\mathrm{CH}_{3} \mathrm{C}-\mathrm{O}$ protons. The $4 \mathrm{H}$, triplet at $3.9 \mathrm{ppm}$ is due to the ethylene bridge protons whereas the singlet at $4.91(2 \mathrm{H})$ is attributed to the vinyl hydrogens. This is related to the broad band at 10.60 integrated for $2 \mathrm{H}$ which likely arises from the strongly hydrogen-bonded enolic form. This shows that in this solvent the structural form of this ligand is strongly hydrogen-bonded.

Table 5. Proton resonance data for the ligand in $\mathrm{CDCl}_{3}$ relative to tetramethylsilane as internal reference

\begin{tabular}{cc}
\hline Signal, ppm & Assignment \\
\hline 10.60 (br) $2 \mathrm{H}$ & Sym. O-H, $N-N$ proton, - hydrogen bonded \\
$4.91(\mathrm{~s}) 2 \mathrm{H}, 3.38$ & Sym. $-\mathrm{CH}=$, methylene protons \\
3.39 (t) $4 \mathrm{H}, 2.30$ & $-\mathrm{CH}_{2}-\mathrm{CH}_{2}$, methylene bridge protons \\
1.91 (d) $12 \mathrm{H}, 1.82$ & Sym. $\mathrm{CH}_{3} \mathrm{C}-\mathrm{O}$ Non-equivalent \\
\hline Legend: $d=$ doublet $;=$ triplet $;$ br $=$ broad; $s=$ singlet $;$ Sym $=$ synthesized
\end{tabular}

Most of the complexes showed single and broad absorption. This has been attributed to paramagnetic effect. This effect is observed in all the complexes implying that they likely have the same structural forms.

\section{References}

1. Okafor E C, Talanta, 1980, 27, 887-891.

2. Adel S O, Adel E M, Medhat A S and Alaa E A, Bull Chem Technol Macedonia, 2005, 24(1), 11-19.

3. Holtzclar H F, Colman J P and Alire R M, J Am Chem Soc., 1958, 80, 1100.

4. Weinstein J and Wyman G M, J Org Chem., 1958, 23, 1618.

5. Witkop B, J Am Chem Soc., 1956, 78, 2873.

6. Dudek G O and Holm R H, J Am Chem Soc., 1961, 83, 2099.

7. Dudek G O and Holm R H, J Am Chem Soc., 1962, 84, 2691-2696.

8. Corden J P, Errington W, Moore P and Wallbridge M G H, Acta Crystallogr Sect C Cryst Struct Commun., 1997, 53(4), 486-488.

9. Roger M S and Tony G H, Anal Chim Acta, 1985, 166, 271-276.

10. McCarthy P J and Martell A E, J Am Chem Soc., 1956, 78, 264.

11. Agget J and Richardson R A, Anal Chim Acta, 1969, 1970, 50, 269-275.

12. Nwabue F I and Okafo E N, Talanta, 1992, 39(3), 273-280.

13. Shijo Y, Sato H, Uehara N, and Aratake S, Analyst, 1996,121, 325-328.

14. Shijo Y, Uehara N, Kudo T, and Aratake S, Anal. Sci., 1994, 10, 951-953.

15. Horspool L J I and McKellar Q A, Biomed Chromatogr., 1991, 5(5), 202-206.

16. Sarma L S, Kumar J R, Reddy J K, Thriveni T and Reddy V A, J Braz Chem Soc., 2006, 17, 463-472.

17. Uzoukwu B A, Gloe K and Duddeck H, Radiochim Acta, 1998, 83, 69-74.

18. Edmund K, Grzegorz R, Waldemar N, Marek K and Kinga S, Polyhedron, 2003, 22, (7), 1009-1018.

19. Agarwal K R, Singh L. \& Sharm D K, Bioinorg Chem Applications, 2006, 4, 1-10.

20. Uden P C and Henderson D E, J Chromatogr., 1974, 99, 309.

21. Gaye M, Sarr O, Sall S A, Diouf O and Hadabee S, Bull Chem Soc Ethiopia, 1997, 11(2), 111-119. 


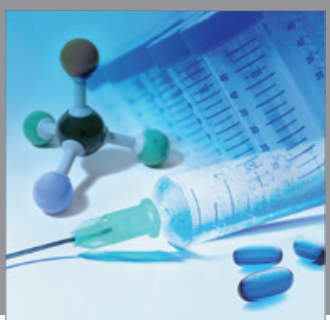

International Journal of

Medicinal Chemistry

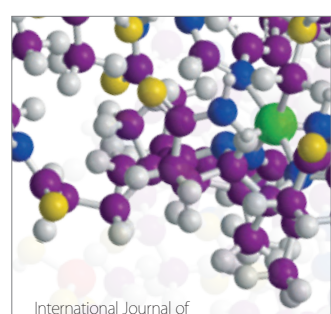

Carbohydrate Chemistry

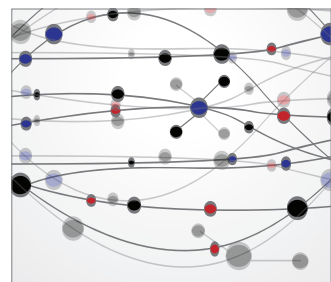

The Scientific World Journal
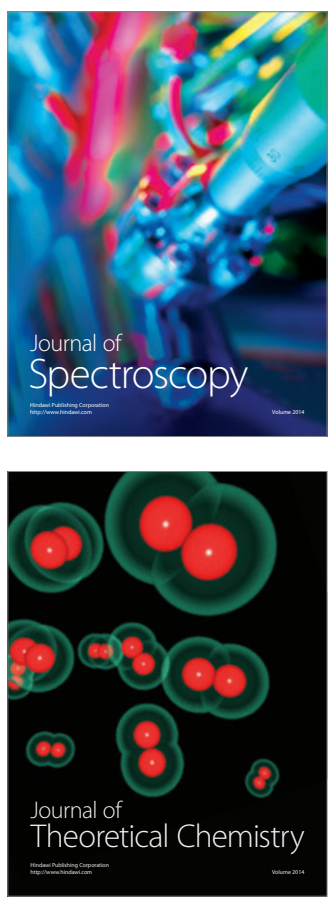
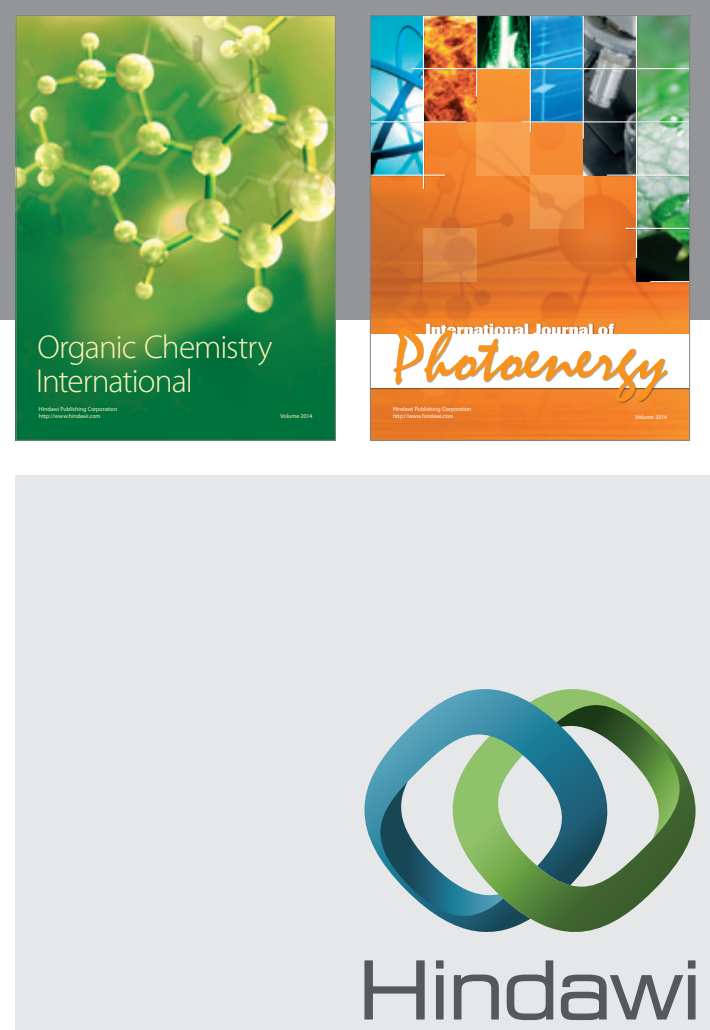

Submit your manuscripts at

http://www.hindawi.com
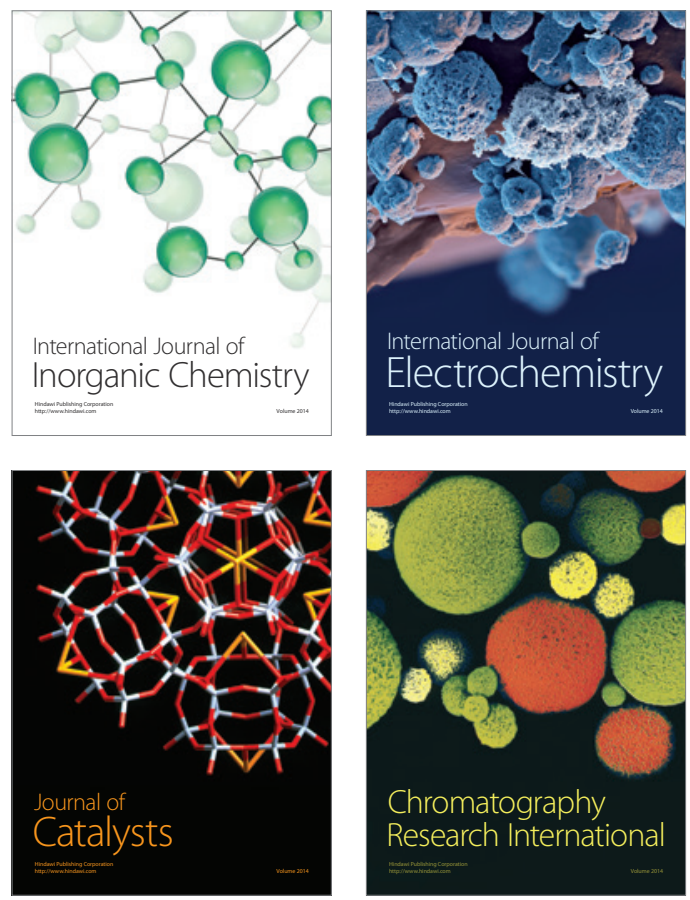
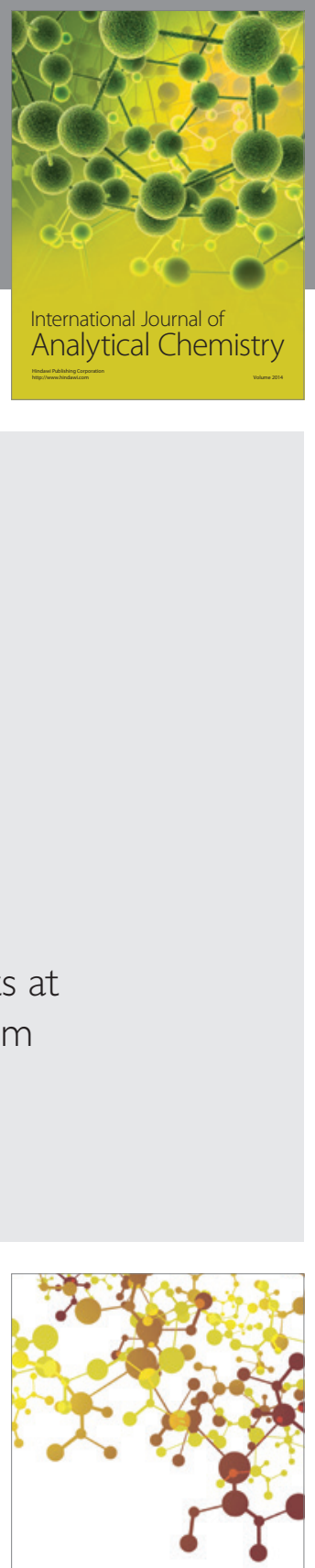

Journal of

Applied Chemistry
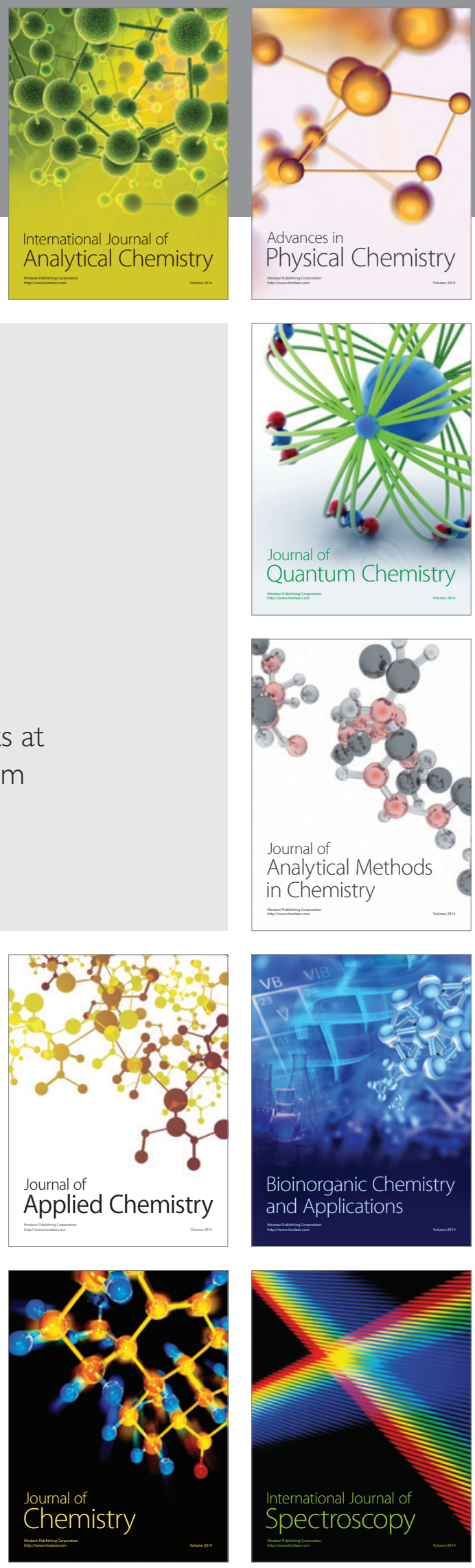